\title{
PRESENZE GENOVESI A SIVIGLIA NELLA SECONDA METÀ DEL QUATTROCENTO
}

\author{
Silvana Fossati RaITERI \\ Universitd di Genova (Italia)
}

La presenza dei mercanti genovesi in Andalusia ed in particolare a Siviglia risale come e noto al secolo XIII ${ }^{1}$ contestualmente alla riconquista castigliana dei territori della regione occupata dagli arabi. E' cospicuo l'aiuto fornito in questo frangente e adeguata la ricompensa da parte del sovrano castigliano. Si trattava di aiuto in combattimento, ma anche in denaro grazie all'attività commerciale dei mercanti genovesi e della Liguria in generale. Infatti il commercio praticato dai genovesi andava dal Levante (con le basi coloniali stabilite in modo definitivo a partire dal trattato del Ninfeo del 1261) all'estremo occidente del Mediterraneo: Siviglia appunto, Cadice, San Lucar de Barrameda.

In territorio castigliano $\mathrm{i}$ genovesi investirono nel prestito gran parte della loro disponibilità finanziaria che avrebbe avuto ulteriori sviluppi nei secoli successivi, favorita dalla successiva acquisizione della residenza (spesso divennero estantes).

Essi furono gia allora finanziatori delle spese militari, ma contribuirono anche molto al ripopolamento e alla ristrutturazione economica. La loro abilità di mercanti li rendeva infatti indispensabili intermediari dei traffici e quindi adatti a favorire l'incremento della produzione agricola ed artigianale, che ne costituiva il punto di partenza.

E' significativo a questo proposito che tra i privilegi loro accordati fosse espressamente citato il commercio dell'olio, che interessava la nobiltà

\footnotetext{
'G.L. FANTONI, L'insediamento genovese a Siviglia nei secoli XII e XIII: aspetti socioeconomici, "Nuova Rivista Storica", LXVII (1983), pp. 60-86.

"Anurario de Estudio Medievales", 24 (1994)
} 
proprietaria dei terreni ove si coltivava l'ulivo². D'altronde i genovesi avevano da sempre mantenuto i traffici con i paesi musulmani, sia in Oriente sia in Occidente, come documentano i trattati stipulati con tali popolazioni a partire dal secolo XII e riconfermati successivamente ${ }^{3}$. Pur appoggiando la guerra di reconquista di Ferdinando III e dei monarchi successivi, non fecero scelte di parte quando si trattava di rapporti economici, e proprio questo li rese preziosi poiche garantivano la continuazione degli scambi tradizionali e il loro ampliamento su basi internazionali, in definitiva assecondando gli interessi della Castiglia e dei suoi sovrani.

Questa politica è all'origine delle concessioni e dei privilegi a favore dei genovesi di Ferdinando III $^{4}$ applicabili non solo ai genovesi di Siviglia, ma di qualunque terra del Regno di Castiglia e León e non revocabili da parte dei successori.

Nei secoli XIV e XV i rapporti commerciali sembrano intensificarsi in particolare con la Catalogna prima e, in seguito alla crisi che interessa Barcellona durante il secolo XV, con Siviglia e Cadice, nella nuova prospettiva occidentale, a causa della situazione critica creata in Oriente dalla pressione turca con la successiva caduta di Costantinopoli. In effetti accanto ai trattati di pace stipulati tra Genova e il Regno catalano-aragonese nel secolo $\mathrm{XV}^{5}$, a partire dal $1403^{6}$ fino al 1455 , sotto i regni di Martino e di Alfonso, si inserisce anche il trattato del $1423^{7}$ stipulato tra il re di Casti-

\footnotetext{
${ }^{2}$ A. COLIANTES DE TERÁN SÁNChEZ, Mercaderes genoveses, aristocracia sevillana y comercio del aceite en el siglo XV, "Tra Siviglia e Genova: notaio, documento e commercio nell'età colombiana", Milano, 1994, pp. 347-359.

${ }^{3} \mathrm{Cr}$. P. LISCIANDRELU. Trattati e negoziazioni politiche della Repubblica di Genova (9581797), "Alti della Socictà Ligure di Storia Patria", n. s. I (1960), reg. 294, 320, 377, 788, 831, 893; R.S. LÓPEZ, Storia delle colonie genovesi nel Mediterraneo, Bologna, 1938, p. 26; G. PISTARINo, Genova e ii Maghreb nel secolo XII, "Aspetti storici di un'amicizia mediterranea", Milano, 1982, pp. 23-68.

4I. GONZALEZ GALJjGO, El libro de los privilegios de la nación genovesa, "Historia, Instituciones, Docuinentos", I (1974), pp. 288-292.

${ }^{5} \mathrm{Cf}$. S. FossatT RAJTER, I trattati tra Genova e Spagna nel secolo XV, "La Spagna nell'età di Colombo", Genova, 1995, a cura di G. AIRAIDI e S. FoSSA in RaItT:RI.

"La pace del 1403 i stata pubblicata da P. Boraruid. y Mascaro, "Codoin", I (1847), pp. 4-24. La pace del 1413 da J. CAMARI:NA MAHIQUFs, Tratados de paz entre Aragón y Génova en 14/3, Valencia, 1953. La pace del 1417 da S. FOSSA'T7 RAITIRI, La pace del 1417 tra la Repubblica di Genova e il Regno di Aragona, "Saggi c Docuinenti", I, Genova, 1978, pp. 454-514. Per le altre paci si veda P. Liscianidri:II, cit. passion.

'Pubblicato da Maria Cristina LUCleko, II trattato del 1423 ira Giovanni II di Castiglia e la Repubblica di Genova, "Saggi c Documenti", VI (Genova, 1985), pp. 307-336.
} 
glia e il Comune di Genova. Rispetto agli altri trattati dello stesso periodo, sottoscritti con il regno catalano-aragonese, per esempio nel $1428^{8}$, questo col re di Castiglia dimostra che Genova viene trattata con dignità di stato autonomo, mentre si trova in realtà sotto la dominazione viscontea: segno che la presenza genovese nel territorio castigliano è rilevante, tanto da condizionarne anche la politica, sottovalutando che la parte contraente avrebbe dovuto essere il duca di Milano, che la occupava a tutti gli effetti e ne era signore.

I buoni rapporti col re di Castiglia stavano a cuore ovviamente anche al comune, ed infatti una delibera del governo e del consiglio degli Anziani ${ }^{9}$ del 24 giugno 1423 stabilisce che i consoli dei genovesi a Siviglia, col consenso dei mercanti che ivi si trovano, possono impiegare fino a $\mathbf{3 0 0 0}$ fiorini o $\mathbf{3 7 5 0}$ lire genovine, ricavandole da gabelle o tassazioni, per le spese che si dovranno sostenere nel corso delle trattative per la stipulazione della pace con il re di Castiglia, il che avverrà nel novembre dello stesso anno.

A tale tine l'll settembre 1423 i consoli dei genovesi in Siviglia deliberano di imporre un drictus dell' $1,5 \%$ su tutte le merci di genovesi o distrettuali del Comune di Genova. Il provvedimento interessa le merci esportate via terra, mare o fiume dal regno di Castiglia verso le città di Murcia, Cartagena, i loro vescovadi e tutti i luoghi e porti di Biscaglia e viceversa. La riscossione dell'imposta viene assegnata con asta pubblica a Giannotto Salvago al prezzo di 610 doppie moresche, da pagare ai consoli e al massaro della loggia. In caso di mancata ratifica da parte del governo genovese il Salvago dovrà ricevere 15 doppie ogni 100 del pagamento pattuito a Siviglia se II risiedono i mercanti, oppure a Genova valutando i cambi di Siviglia.

Sottoscrivono il documento $\mathrm{i}$ consoli ${ }^{10}$ Antonio de Franchi Luxardo e Andalo Lomellini e sedici mercanti, tra cui figurano Cattaneo, Doria, Grimaldi, Lomellini, Spinola e Cristoforo de Chiavari.

\footnotetext{
${ }^{8}$ Il trattato del 1328 d tuttora inedito. Si conserva, per la parte catalana, in ACA, Cancilleria, 2646, cc. 52 r. -72 v.; per la parte genovese, in ASG, Libri iurium reipublice lanuensis III, cc. 14-22 v.

${ }^{9}$ ASG, Diversonum, 507, c. 104 r.-105 v.; ASG, Diversorum, 507, c. 103 v.-106 r.

${ }^{10} \mathrm{Si}$ dimostra necessaria la lom presenza per imporre tributi ed anche per sottoserivere gli atti pubblici.
} 
La delibera in questione viene ratificata lo stesso giorno in Siviglia $^{11}$. Il mercante che ho citato espressamente Cristoforo de Chiavari ${ }^{12}$ e lo stesso che nel 1438, in una carta pubblica rogata in Siviglia, rinuncia a favore del fratello Gerolamo alla proprieta di una casa situata in Genova nella zona di residenza dei Franchi ${ }^{13}$.

Si trattava di due fratelli Boccanegra Chiavari legati alla vita commerciale sivigliana, tanto che Cristoforo viene definito estante in Siviglia nel 1438, quando sono passati quindici anni dal documento citato precedentemente, in cui compare come teste insieme agli altri mercanti genovesi di Siviglia. I due fratelli Gerolamo e Cristoforo Boccanegra, presenti a Siviglia nel 1438, avevano nella città interessi comuni, probabilmente come esportatori di grano e di coloranti, legati ai Rivarolo ${ }^{14}$.

Ma solo Cristoforo rimase stabile nella citta andalusa, mentre il fratello mantenne i legami con Genova ed in particolare la proprietà della casa, sita nella zona di residenza dei Boccanegra, per la cui eredità nel 1465 ci sono contestazioni e si richiede l'atto notarile cui ho fatto riferimento sopra.

Comunque nel corso del XV secolo i legami economici tra le due città sono assai forti e si intensificano in seguito alla crisi che attraversa Barcellona; se consideriamo la documentazione dei protocolli notarili, conservata ora nell'Archivo historico e gli atti del Cabildo del Consejo di Siviglia ${ }^{15}$ possiamo constatare che $i$ genovesi erano ben presenti nella vita economica della città e nel fervore che dovette accompagnare le imprese di Colombo verso il nuovo mondo.

\footnotetext{
"ASG, Diversonum, 507, c. 103 v.-106 r.

${ }^{12}$ La coincidenza del personaggio è quasi ccrta, sopra tutto se si tiene conto degli altri mercanti che sottoscrivono il documento. Per quanto riguarda l'omissione del cognome Boccanegra, si veda la nota seguente.

${ }^{13} \mathrm{Cf}$. S. FossATI RATER, Una carta pubblica tra gli alti di Giovanni da Voltaggio, "Saggi e Documenti", II, Genova, 1981, pp. 368-385.

${ }^{14} \mathrm{Nel} 1439$ la filiale di Bruges della Compagnia Borromeo invid a Siviglia presso Giacomo Rivarolo una partita di fustagni e una di robbia: Archivio Borromeo, Mastro di Bruges, f. 351 v.- 352 r., cit. da P. MAINONI, Mercanii lombardi tra Barcellona e Valenza nel Basso Medioevo, Bologna, 1982, p. 97, n. 51 e G. BISCARO, Il banco Filippo Borrorneo e $i$ compagni di Londra (1436-1439), "Archivio Storico Lombardo", XL (1913), pp. 37-126, 283-385.

${ }^{15} \mathrm{Cf}$. J. BONO-C. UNGUETI BONO, Los protocolos sevillanos de la época del descubrimiento, Sevilla, 1986 e M.J. SANZ FUENTES-M.I. SIMÓ RODRIGUEZ, Catálogo de documentos contenidos en los libros del cabildo del concejo de Sevilla, Sevilla, 1975.
} 
Sulla base della documentazione citata, a cominciare dai documenti del Cabildo del Consejo di Sivigliaa ${ }^{16}$, tratteggio ora come questi mercanti erano inseriti nella vita della città. Per esempio il vecino della città, e percio con pieni diritti civili, Juan Ortiz de Juanguren si lamenta davanti al Consejo di Siviglia di essere stato incarcerato e poi portato alle atarazanas $^{17}$ dal mercante genovese Giovanni Falamonica ${ }^{18}$ che ha approfittato del favore che godeva presso alcuni nobili (10 aprile 1448) e l'anno dopo (31 marzo 1449) lo stesso Juan Ortiz chiede di essere scarcerato per provare di essere stato calunniato dal mercante genovese ${ }^{19}$. La questione non e ancora risolta il 6 luglio 1450 quando Catalina Ferrandez, anch'essa vecina della collación di S.Salvador, moglie di Ortiz, informa che il marito da due anni $e$ in carcere ed ora si trova a Niebla per certe doppie richieste dal genovese Falamonica ${ }^{20}$. La donna chiede che il marito sia riportato nella città e sia fatta giustizia. Tuttavia sembra che le autorità propendano a favorire il genovese, che non risulta essere vecino, ma solo mercante, anche se in buoni rapporti con alcuni nobili della città, e questo conta.

Dal documento risulta che $i$ consoli genovesi Marco Cattaneo e Giacomo Salvago avevano condannato Juan Ortiz al pagamento di 1400 doppie, e che quest'ultimo avesse impugnato la sentenza davanti all'alcalde mayor Pedro de Guzmán. Si desume che i consoli della comunità genovese esercitassero un certo potere anche nelle cause in cui la controparte era un vecino sivigliano, come in questo caso. Per quanto riguarda le attività commerciali, analogamente a quanto era sempre avvenuto nel regno catalano-aragonese, anche in Castiglia ci sono tentativi del sovrano di controllare e, se possibile, monopolizzare il commercio. Cosl un documento del 18 settembre 1452 del re Giovanni II dispone ${ }^{21}$ che tutti i mercanti spagnoli, genovesi, veneziani e fiorentini, ossia quelli che erano soliti praticare commerci nella zona, debbano caricare le loro mercanzie dirette alle Fiandre nella caracca del re che va a Santander da Siviglia. E ancora, sempre in

\footnotetext{
${ }^{16}$ AMS (Archivo Municipal de Sevilla)

${ }^{17}$ Si tratta degli arsenali navali; successivamente fu imprigionato a Niebla.

${ }^{18}$ AMS, AAC, 1448, fol. 24.

${ }^{19}$ AMS, BAC, 1449, fol. 6.

${ }^{20}$ AMS, AAC, 1450, fol. 53.

${ }^{21}$ AMS, BAC, 1452, fol. 127-128.
} 
questo periodo ${ }^{22}$, abbiamo esempi di mercanti genovesi (come Galeazzo Cicala) che trasportano merci da Genova all'Inghilterra, cui si vieta all'andata come al ritorno di entrare in «río Sibilie» e di toccare Cartagena. Un documento del 16 febbraio 1459 e un altro del 19 febbraio informano ${ }^{23}$ il Concejo di Siviglia che Giovanni Centurione, genovese residente in Siviglia, nel viaggio verso Lebrija è stato derubato e portato al castello di Corte (il furto ammonta ad un valore di 20000 maravedis, tra enriques e reali, oltre a 40 doppie $^{24}$ ): certamente denaro da investire in mercanzie; il secondo documento, in particolare, è la richiesta di giustizia da parte dei consoli dei mercanti genovesi per il loro compatriota e l'accusa di non avere tenuto fede al seguro del re per i mercanti genovesi. Si documenta quindi la posizione che essi avevano acquisito in Siviglia con la possibilita di commerciare più o meno liberamente, sulla base di accordi con la Corona castigliana e di salvacondotti.

Ancora in un documento del 16 ottobre $1454^{25}$ i consoli e i mercanti genovesi richiedono la libertà per alcuni di loro che erano stati imprigionati dagli esattori delle imposte, forse perchè le avevano evase. Nonostante questi incidenti i genovesi avevano posizioni di privilegio, come dimostra la richiesta dei corredores (agenti) della dogana il 18 ottobre 1454 , che non vogliono sia loro concesso di esercitare la funzione di corredor senza il relativo permesso ${ }^{26}$. Evidentemente $\mathrm{i}$ genovesi erano ritenuti concorrenti temibili tanto più se potevano essere occupati in tale funzione di notevole importanza senza dovere richiedere il permesso preventivo, come sembrerebbe ovvio.

Ma essi tuttavia subivano anche soprusi, come testimonia l'11 novembre 1454 Sancho Draz de Medina, che a nome dei consoli e dei mercanti genovesi di Siviglia ${ }^{27}$, informa il Consejo dei furti e danni che ricevevano andando da San Lucar a Siviglia, da parte di alcuni vecinos di quel porto.

\footnotetext{
${ }^{22}$ ASG, notaio Obcrto Foglietta, filza 5, n. 9, citato da J. HEERS, Les patrons de navire a Gênes au teinps de la jeunesse de C. Colomb: compétences, expériences, profil, "Atti del IV Convegno Intemazionale di Studi Colombiani (21-23 ollobre 1985)", Genova, 1987.

${ }^{23}$ AMS, AAC, fol. 49.

${ }^{24}$ Così ncl regesto.

${ }^{25}$ AMS, AAC, 1454, fol. 53.

${ }^{26}$ AMS, BAC, 1454, fol. 58.

${ }^{27}$ AMS, BAC, 1454 , fol. 92
} 
E ancora lagnanze vengono fatte dai mercanti genovesi che venivano danneggiati da chi era nella galea patronizada da Baeza, criado di don Martín Guzmán ${ }^{28}$. Quindi troviamo i mercanti genovesi anche in veste di danneggiati ad opera di locali, neppure di alto lignaggio. E' comunque sempre garantita da parte del re la protezione, se è vero che il 15 dicembre 1454 Enrico IV $^{29}$ ordina che sia prorogato il salvacondotto che il padre Giovanni II aveva concesso ai mercanti genovesi residenti in Siviglia il 30 giugno 1451. Anche il nuovo sovrano ritiene opportuno continuare la politica di alleanza con i genovesi che contribuiscono ad attivare i commerci con la Castiglia, seguendo la tradizione.

Infatti essi intrattengono rapporti commerciali con altri mercanti, ad esempio Barnaba Sisto $^{30}$ il 24 ottobre 1453 chiede al Consejo di poter vendere liberamente coloranti che tiene in casa sua $\mathrm{e}$ in casa del milanese Antonio Pasaliago, e che gli sono stati inviati per mare. Come e noto Genova $e$ Milano avevano notevoli industrie di lavorazione e tintura dei panni, che davano vita ad un cospicuo commercio di panni e di materie coloran$\mathrm{ti}^{31}$.

Anche in altre attività industriali e commerciali della città i genovesi sono inseriti o tentano di inserirsi, come nella lavorazione dell'argento: il 26 ottobre 1467 i consoli e i mercanti genovesi informano il Consejo ${ }^{32}$ che gli argentieri della citta avevano ripartito tra di loro la rendita dell'imposta dell'oro e dell'argento che la citta appaltava, evidentemente ritenevano di avere diritto alla compartecipazione. Il documento è un estratto di un atto capitolare, e quindi ufficiale.

I consoli dei mercanti genoves $i^{33}$ il 2 luglio 1470 negano di avere rotto la promessa di osservare la proibizione di commerciare grano e affermano che la loro caracca, che si trova a Cadice, non porta grano, come è stato detto erroneamente, ma tonno e cuoio. Il commercio del tonno era fiorente già a metà del secolo, come testimonia un documento del febbraio

\footnotetext{
${ }^{28}$ AMS, BAC, 1454, fol. 98.

${ }^{29}$ AMS, BAC, 1455, fol. 49-50.

${ }^{30}$ AMS, AAC, 1453, fol. 4.

${ }^{31} \mathrm{Cf}$. J. HEERS, Les patrons, cit., p. 70.

${ }^{32}$ AMS, CAC, 1467, fol. 31.

${ }^{33}$ AMS, AAC, 1470, fol. 33.
} 
$1458^{34}$. Per quanto riguarda il grano, e noto che nei periodi di carestia era previsto l'obbligo di importare l'equivalente in grano delle altre merci esportate, in particolare l'olio.

Nella commercializzazione di quest'ultimo prodotto $i$ genovesi avevano grandi interessi fin dal periodo del citato privilegio di Fernando III, che li favoriva espressamente. Poiche si trattava di un prodotto assai diffuso, ma in mano alla nobiltà locale, che ne controllava la produzione fin dalla coltivazione dell'ulivo, si verificava una convergenza di interessi e i primi acquirenti e successivi mercanti erano sempre i genovesi. Si fa eccezione nel 1478 quando $i$ cavalieri «que tenemos olyvares y heredades en el Axarafe desta cibdad» lamentano che i genovesi, invece di limitarsi al trasporto dell'olio, non lo caricano, ma lo tengono, cioe diventano regatones, ovvero incettatori $i^{35}$. Naturalmente protestano i genovesi rivendicando il loro privilegio di comprare e vendere l'olio, come fanno i firmatari della petizione, mentre ribadiscono fermamente «somos previllejados e tenemos previllejo usado, guardado e confirmado por los reyes pasados». Tutto questo avviene durante il soggiorno a Siviglia dei re Cattolici, che si fermarono appunto nel 1477-78 e dovettero riportare l'ordine dopo le guerre civili. Negli anni immediatamente precedenti questo periodo la situazione dei genovesi era diventata meno sicura, e perciò $i$ consoli informano che la Signoria di Genova ha armato navi per difendere i suoi mercanti dalle razzie troppo frequenti da parte di corsari di varie nazioni (13 aprile 1472)$^{36}$. Ricordiamo che per la Castiglia e il periodo della lotta finale con il regno moro di Granada e che Genova si trova sotto il dominio sforzesco. Tuttavia anche in questa circostanza, come era accaduto nel trattato di pace con la Castiglia, sopra citato, viene sempre ricordata come Signoria di Genova, la potenza che da sempre è presente sulle rotte commerciali castigliane.

\footnotetext{
${ }^{4}$ Cf. ASG, notaio Bartoloneo Risso, filza 5, in cui si riferisce che un lembo ha caricato nelle tonnare 65 barili di tonno, conservato sotto olio, e trasferito a Cadice nella nave di Demetrio Cattaneo per essere consegnato a Savona, città della Rivicra, in rapporti commenciali con la penisola iberica. Cf. J. HrEers, La Riviera di Ponente el les grandes découvertes maritimes: économies el sociétés "V Convegno Storico Savonese. 'L'ctà dei Della Rovere'". "Alti c Memorie. Società Savonese di Storia Patria", n. s., XXV (Savona, 1989); e C. VARALDO, Savona nel Secondo Quattrocento. Aspetti di vita economica e sociale, "Savona nel Quattrocento e l'istituzione del Monte di Pietà", Savona, 1980.

${ }^{35}$ AMS, Sección 10, 1478-VIII-19, pubblicato da A. COLlanteS DI: TeRÁN SÁNCHEZ, Mercaderes genoveses, cit., p. 356.

${ }^{36}$ AMS. AAC, 1472, fol. 60.
} 
Il 3 febbraio 1474 sempre la Signoria di Genova lamenta i maltrattamenti che ricevono $\mathrm{i}$ suoi mercanti ${ }^{37}$, minacciando la rottura delle tradizionali relazioni amichevoli.

Un ultimo documento ${ }^{38}$, non datato, riferisce che gli appaltatori delle rendite delle mercanzie del porto di Siviglia si lamentano che Pero Ibañez, capitano della caracca del re si impadronisce di tutte le merci, tanto che $i$ genovesi si sono ritirati e hanno ormeggiato le navi a Sanlucar e a Cadice. La loro presenza doveva essere importante se si chiede che veng 2 allontanato il capitano del re, a vantaggio dei genovesi e in generale dei commerci, che a loro sono riferiti.

I mercanti genovesi garantiscono da sempre anche i collegamenti con il Nord, in particolare con l'Inghilterra e le Fiandre e lo spostamento della loro base da Siviglia a SanLucar e a Cadice puo ripercuotersi negativamente per l'economia castigliana.

Sembra dai documenti esaminati che in un primo tempo i mercanti, compreso i genovesi, fossero obbligati a trasportare le merci che andavano nelle Fiandre sulle navi del re, come prevedeva la disposizione del documento sopra citato del 1452, emessa dal re Giovanni, ma successivamente anche per i soprusi denunciati, si teme che preferiscano rimanere nei porti di Cadice e SanLucar e si lascia loro libertà di caricare nelle loro navi, come vedremo in documenti specifici.

Negli atti ufticiali del Cabildo ho trovato mercanti come Centurione, Falamonica e Sisto, inseriti nella vita economica della citta legati alla nobiltà e protetti dalla monarchia.

I documenti notarili che citero nel seguito sono della seconda meta del secolo e testimoniano come i genovesi avessero parte attiva in molteplici attività, legati ora a Siviglia, ora al commercio che da ll si diramava.

Il 12 agosto $1472^{39}$, un mercante inglese vende a Geronimo Grimaldi 500 pezze di panno al prezzo di undici doppie una per l'altra, dilazionate in ventun mesi, con sconto se pagate prima di detto termine.

Un documento del 12 e 13 novembre $1489^{40}$ attesta la partecipazione dei mercanti di Genova alle spese per l'assedio di Baza, che come

\footnotetext{
${ }^{37}$ AMS, BAC, 1474, fol. 17.

${ }^{38}$ AMS, BAC, siglo XV, fol. 661,

${ }^{39}$ AHP, Oficio 23, Francisco Sánchez 23.2, fol. 242 r.-v.

${ }^{40}$ AHP, Oficio 3, Antón Ruiz. de Porras, 3.1, fol. 251 v.-252 v.
} 
noto fu la più costosa azione di guerra per la conquista di Granada ${ }^{41}$. L'assedio duro dal 15-18 giugno fino al 4 dicembre 1489. Quindi il documento cui mi riferisco e emanato nella fase cruciale e determinante della guerra: il bachiller Alonso Tellez nella loggia dei genovesi richiede ufficialmente ai consoli Bernardo Grimaldi e Andrea Odone, questi a nome di Rufo Doria e ad altri mercanti il pagamento di un conto di maravedís gia richiesto in una lettera della regina, notificata da dieci giorni. In questa occasione viene gia fatta la ripartizione tra i mercanti della cifra richiesta e si esige il saldo entro tre giorni, sotto pena di confisca dei beni. Il 18 novembre ${ }^{42}$ lo stesso Tellez, come esecutore della regina, confisca differenti mercanzie in esecuzione della ripartizione effettuata. I nomi dei mercanti sono tra i più noti: Ruffo e Giuliano Doria, Giovanni Lomellini, Celino Cattaneo, Martino Pinelli, Francesco Rivarolo, Francesco e Angelo de Negro, Pellegro Usodimare, Bernardo Grimaldi, Luigi Tarigo e Baliano Salvago. Interessante è anche l'elenco delle mercanzie di cui si dà l'equivalente in denaro: si tratta sostanzialmente di tre pezze di raso nero, tre pezze di velluto nero, cinquanta balle di carta, quattro pezze di velluto carmesi, una pezza di velluto villocado e mezza di carmesl colorato, cinque pezze di velluto nero (il pezzo più consistente è attribuito a Francesco Rivarolo), tre pezze di raso nero, cinque quintali di indaco, una pezza di raso nero di sedici acediras morische, una pezza di velluto carmesl, sei balle di un tessuto di seta ${ }^{43}$, una pezza di carmesl e cinque pezze di velluto nero e venticinque balle di carta straccia, tre pezze di velluto nero, due pezze di velluto nero, ed infine una pezza di carmesl doblado. La cifra totale risulta notevole, ma interessa anche il genere di prodotti che documenta la consistenza del commercio di tessuti preziosi di cui i genovesi sono grandi esportatori; il documento citato, oltre a presentare i genovesi come sostenitori di spese di guerra, dimostra la loro importanza come mercanti di prodotti pregiati e costosi, interessanti l'economia castigliana.

Un altro documento dell' 11 marzo $1491^{44}$ indica Francesco PineIli, il famoso mercante legato a Cristoforo Colombo, come fedele esecutore p. 601 .

${ }^{41}$ Cf. L. SuÁrez Fernández, Historia de España. Edad Media, Madrid, Gredos, 1970,

${ }^{42}$ AHP, Ofício 3, Antón Ruiz de Porras, 3.1, fol. 262 r.-264 r.

${ }^{43}$ Nella trascrizione di Ungueti Bono, cit., risulta "anafra", que potrehbe essere invece "anafalia".

${ }^{4}$ AHP, Oficio 4, Francisco Segura, 4.1, fol. 444 r.-v. 
del tenente e assistente di Siviglia, a cui si rivolge lo scrivano pubblico di Siviglia, per un requerimiento. La questione non si risolve ed ancora il 18 febbraio $1495^{45}$ si richiede l'appello per la causa dovuta all'eccessiva richiesta fatta dagli scrivani per le loro tariffe e la notifica e fatta ancora a Francesco Pinelli. In questo caso, ma si tratta di cosa nota, un genovese è inserito nella vita pubblica sivigliana. Sempre Francesco Pinelli il 16 luglio $1493^{46}$ riceve dai re Cattolici l'incarico di distribuire ai vari conventi di Siviglia, Cordoba, Ecija e Jérez le elemosine del defunto duca di Cadice (; tonni delle almadrabas), e viene chiamato giurato e fedele esecutore di Siviglia.

In un altro documento ${ }^{47}$ il libraio Urbano e sua moglie, vecinos di Siviglia e della collación di Santa Maria, dove risiedevano i genovesi, il 7 luglio 1494 riconoscono nei riguardi del libraio Nicoloso un debito di 8.000 maravedis prestati loro per pagare il mercante genovese Teramo, residente nella città, il pagamento sarà effettuato entro quattro mesi e in pegno si danno dei libri che sono elencati, classici e una bibbia manoscritta. Si documenta in questo caso l'intervento genovese nel commercio dei libri, per una somma consistente e con una certa varietà, tra classici latini e testi religiosi e scientifici, tutti stampati ed inquadernati, come si specifica. Il 19 ottobre $1495^{48}$ il mercante genovese Guido Lavezzari conferisce procura al farmacista Geronimo Varo per riscuotere i crediti derivanti dall'importazione di libri da Venezia. Ancora una volta il mercante genovese si inserisce nella attività commerciale e fà da tramite con mercanti di altre nazioni, presenti nella città.

Due documenti del $1498^{49}$ vedono la richiesta fatta a Bernardo Grimaldi di pagare una lettera di credito di 60000 maravedís a Martín de Almonte da parte di Cristoforo Colombo; nel secondo documento si tratta di una lettera di cambio di 961 doppie e un quarto kescripta con letra genovisca ... declarada en castellano», presentata a Giovanni Lomellini dal mercante valenziano Geronimo Alegre. Siamo ormai in epoca colombiana e

\footnotetext{
${ }^{45}$ AHP, Oficio 4, Francisco Segura, 4.2, fol. 14 r.-16 v.

${ }^{46} \mathrm{AHP}$, Oficio 5, Gonzalo Bernal de la Becerra, 5.16, fol. $51 \mathrm{r}$ - $\mathrm{v}$.

${ }^{47}$ AHP, Oficio 4, Francisco Segura, 4.1, fol. 662 r. -663 r.

${ }^{48}$ AHP, Oficio 4, Francisco Segura, 4.2, fol. $419 \mathrm{r}$.

${ }^{49}$ AHP, Oficio 9, Luis García de Celada, 9.30, fol. 139 v. e Ofício 5, Gonzalo Bemal de la Becerra, 5.28, fol. 72 v. -73 r.
} 
i mercanti genovesi vengono direttamente coinvolti nelle rotte atlantiche, nel caso di Bernardo, da Colombo stesso, nell'altro da mercanti valenziani di Siviglia.

Resta pertanto documentata la presenza genovese nei nuovi interessi che ha risvegliato l'impresa di Colombo, ma rimane sempre importante il commercio in area europea, in particolare nel Nord: in un documento del primo giugno $1499^{50}$ il maestro della nave Santa Maria, ormeggiata nel porto di Moguer, noleggia la stessa a Stefano Gentile, vecino di Moguer e a Gonzalo Suarez, mercante, vecino di Siviglia della collación di Santa Maria, dove solevano risiedere i genovesi. Il noleggio prevede un carico di vino e altre mercanzie per cinquantacinque tonnellate a Stefano Gentile e ventinove a Gonzalo Suarez. La destinazione è il porto di Tagafuerta in Irlanda e in secondo lúogo Galway ${ }^{51}$, al prezzo di nove corone e tre quarti per tonnellata, della moneta locale.

Dalla documentazione esaminata risulta chiara la posizione che $i$ mercanti genovesi avevano in Siviglia: i privilegi loro concessi a partire da Fernado III nel 1251 a distanza di duecento anni si sono conservati ed essi estantes o più spesso vecinos di Siviglia, e quasi sempre della collación di Santa Maria, hanno cariche ufficiali, come nel caso del citato Francesco Pinelli. Inoltre hanno legami economici con la nobilta sivigliana, che li protegge, cosl come la monarchia che concede loro salvacondotti, pur con le dovute eccezioni; come nel caso sopra citato del commercio dell'olio, risolto tuttavia in favore dei genovesi.

Sembra infine interessante rilevare l'ostilità degli agenti della dogana di Siviglia che si oppongono a che i genovesi possano esercitare il loro stesso ufficio: il che testimonia che questi erano attivi come mercanti a tutti i livelli, anche nelle cariche connesse alle attivita commerciali.

Non stupisce quindi il totale.inserimento e la spagnolizzazione di esponenti di famiglie cospicue genovesi in territorio andaluso, che' abbiamo visto: Pinelli, Centurione, Grimaldi, Lomellini.

Ma compaiono anche esponenti di alberghi popolari come i Franchi e i Soprani. Ai primi appartengono i Boccanegra, sopra citati, i Luxardo, i Sauli; ai secondi i Rivarolo, Cassano, Castiglione, che sono originari della Riviera. Si documenta anche l'importanza di Savona, inserita nel commer-

\footnotetext{
${ }^{50}$ AHP, Oficio 3, Juan Ruiz de Porras, 3.3, fol. 50 v. -53 r.

"La trascrizione di Bobo-Ungueti Bono, cit., segnala Tagafuerta con punto interrogativo.
} 
cio spagnolo, come si evince dal documento del 1457 sopra citato. Di Cadice abbiamo parlato in quanto base commerciale, soprattutto nel momento della crisi di Siviglia per le lotte del 1471 tra il duca di Medina Sidonia e il marchese di Cadice, che costarono ai cittadini nuovi tributi. La città rimase isolata «envuelta en los horrores de la guerra civil» ${ }^{32}$ : probabilmente il documento non datato che ho citato si pud riferire a questi anni, dal 1471 al 1474, in cui prevale il marchese di Cadice contro il duca di Medina Sidonia. Le discordie civili furono soffocate dai re Cattolici, senz'a che dovessero ricorrere a metodi drastici e con opera di mediazione tra $i$ due contendenti, affidando il governo della città di Siviglia al conte di Tendilla nel 1475. Nel 1477 entro in Siviglia la regina e qualche mese dopo il re, entrambi rimasero un anno e mezzo, prestarono attenzione alle varie lagnanze e agirono con cautela, mantenendo al loro posto uomini dell'amministrazione precedente.

Gli alcazares furono lasciati in un primo tempo al duca di Medina Sidonia $^{53}$, poi passarono con le atarazanas a Diego de Merlo, cui si deve il cambiamento della politica marittima con l'introduzione delle caravelle, preannuncio della futura politica atlantica castigliana.

La citta di Siviglia a partire da questo periodo è ancora di più in mano ai mercanti ed è appoggiata dai sovrani, tesi a diminuire la potenza dei nobili. Questi a loro volta si appoggiano ai conversos, e si spiega quindi la politica di persecuzione a cui sono soggetti con l'introduzione del Santo Uffizio.

Tuttavia le persecuzioni crearono problemi economici e si giunse a una sorta di composizione: i conversi, grazie alle loro disponibilità economiche, saranno in seguito i protagonisti della politica atlantica ${ }^{54}$; $i$ genovesi rimangono bensl in Siviglia, come abbiamo documentato, ma contemporaneamente si aprono ai nuovi mercati dell'Atlantico: siamo ormai all'epoca di Carlo V e al «siglo de los genoveses».

${ }^{32}$ ALONSO DE PALENCIA, Crónica de Enrique $N$, Década I.85 (BAE, 258 b).

${ }^{53}$ El tumbo de los Reyes Católicos del Concejo de Sevilla, ed. dirigida por R. CARANDE y J. de M. Carriazo, I, Sevilla, 1968, p. 51.

"CF. J. GIL, Sevilla: de feudo nobiliario a puerto de las Indias, "La Spagna", cit. 


\section{RÉSUMÉ}

L'établissement des Génois en Séville, qui eut son commencement au XIII ${ }^{e}$ siecle avec les privileges accordés aux Génois par le roi Fernand III, se développe dans les siècles suivants. Dans cet article on examine des documents des Archives Municipaux et des Archives des Protocoles de Séville de la deuxième moitie du $X^{e}$ siècle, où des marchands génois appartenant aux familles Grimaldi, Doria, Cattaneo, Lomellini, Boccanegra et d'autres participent à la vie économique et politique de la ville. A titre d'exemple, Francesco Pinelli, ami de Christophe Colombo, est cité comme fidele exécuteur de Séville dans un litige entre castillans. Les marchands génois obtiennent encore la confirmation de leurs privilèges, notamment pour le commerce de l'huile. A la fin du siecle, les génois vont ouvrir leurs commerces aux nouvelles routes atlantiques.

\section{SUMMARY}

The genoese settling in Sevilla began in the XIIIth century with the privileges granted by king Ferdinand III, but only in the following century it became substantial. In this article, some documents from the Municipal Archives and from the Archives of the Registers of Sevilla of the second half of the XVth century are examined. In the documents some genoese merchants belonging to important families such as Grimaldi, Doria, Cattaneo, Lomellini, Boccanegra, participate in the economic and political life of the city. An exemple is Francesco Pinelli, friend of Colombo, who is cited like faithful executor of the city in a controversy among citoyens of Sevilla. The genoese merchants had their privileges confirmed, in particular for the commerce of the olive oil. At the end of the century, the genoese merchants extended their trades to the new atlantic routes. 